\title{
Models for Fault Current Limiters based on Superconductor Materials
}

\author{
A. Etxegarai ${ }^{1}$, I. Zamora, G. Buigues, V. Valverde, E. Torres and P. Eguia \\ ${ }^{1}$ Department of Electrical Engineering, Faculty of Engineering of Bilbao, UPV/EHU \\ Alameda de Urquijo s/n, 48013 Bilbao (Spain) \\ e-mail: agurtzane.etxegarai@ehu.eus
}

\begin{abstract}
The present paper introduces the main electric applications of superconducting materials, focusing on Superconducting Fault Current Limiters (SFCL). In recent years, short-circuit current values have increased, mainly due to a higher penetration of distributed generation. Fault Current Limiters (FCL) based on superconductor materials can provide one of the most promising solutions for limiting those fault currents. Nowadays, research focuses mainly on resistive SFCLs, because of size, cost and product maturity. Several modelling approaches for resistive type current limiters, as proposed in the literature, are reviewed hereby.
\end{abstract}

\section{Key words}

Superconductivity, Superconducting Fault Current Limiter (SFCL), modelling, simulation.

\section{Introduction}

Superconductivity is the phenomenon that occurs when materials exhibit zero electrical resistance below a characteristic critical temperature and are perfectly diamagnetic. There is a wide range of materials that present these characteristics. The first materials to show superconducting properties were pure metals at temperatures approaching absolute zero, e.g. Mercury at $4.2^{\circ} \mathrm{K}$. This group of materials later included metal alloys such as Niobium-tin $\left(\mathrm{Nb}_{3} \mathrm{Sn}\right)$ with also critical temperature values near absolute zero. Liquid Helium was required as coolant. Earlier materials are now referred to as low temperature superconductors (LTS), with critical temperatures below $30^{\circ} \mathrm{K}$.

In 1986, researchers at IBM discovered superconductors made from copper oxide ceramics with much higher critical temperature values: High Temperature Superconductors (HTS). The most commonly used materials in early HTS were Bismuth-based, BSCCO, most commonly Bi-2212 and Bi-2223. These materials have come to be known as first generation (1G) superconductors More recently, rare earth-based HTS materials ((RE)BCO) have been developed, known as second generation $(2 \mathrm{G})$ superconductors.
Yttrium-based materials (YBCO) resulted into higher critical temperatures. Many other cuprate superconductors have since been discovered and used for several applications. As an instance, $\mathrm{MgB}_{2}$ was proved to have a transition temperature of $39^{\circ} \mathrm{K}$, ranging between low temperature and high temperature superconducting materials. It is a material that can be produced in long length at competitive costs [1].

The increase in critical temperature has been essential in the development of superconducting materials. LTS needed to be cooled by liquid Helium, while HTS ceramic compounds exhibit superconductivity at higher temperatures. Therefore, materials can be cooled by liquid Nitrogen, which can be liquidized at $77^{\circ} \mathrm{K}$ and is far less expensive. However, ceramic materials are breakable and unductile. As a result, manufacturing superconductor wires is complex. In addition, in order to guarantee superconducting characteristics, conditions must be within critical temperature, current density and magnetic field values.

This paper presents the modelling approaches of resistive type Superconducting Fault Current Limiters (SFCL), which are one of the most promising applications of last generation superconductor materials.

\section{Power system applications of superconductors}

Along with showing zero electrical resistance, the Meissner effect also occurs in superconducting materials; that is, the expulsion of magnetic fields. The first characteristic can be used in high performance power system applications, while the second results into the powerful electromagnets. The present paper focuses on the application of superconductivity in power systems. Main requirements of superconducting materials for this field are high critical temperature, magnetic field and current density, stable operation, ease of manufacturing and low cost. 
As indicated by the most recent reviews in literature, the most promising applications in the field are superconducting cables, SMES (Superconducting Magnetic Energy Storage) and SFCLs (Superconducting Fault Current Limiters). Other promising applications mentioned in literature are rotating machines and transformers.

\section{A. Superconducting cables}

First prototypes of superconducting cables were based on LTS using Helium cooling system. However, nowadays, they have been mostly replaced by HTS cooled by liquid Nitrogen. The manufacturing process and ductility is worse than in LTS cables, but it is less expensive and the cooling system has an easier design. Today, commercially available HTS cables are BSCCO and YBCO. Alternatively, LTS cables are based on $\mathrm{MgB}_{2}$, which has a good performance/cost ratio. Two designs were initially proposed for superconducting cables: warm dielectric design (WD) and cold dielectric coaxial design (CD). Nowadays, the commercially available cables are the cold dielectric cables; where the dielectric of the cable is located inside the cryogenic cold portion of the cable structure.

Most of the HTS cables have been projected for AC systems, achieving a more compacts. HTS cables for DC systems are still in an earlier stage, but with promising results.

\section{B. SMES (Superconducting Magnetic Energy Storage)}

Energy storage technologies are becoming key due to the increasing penetration of renewable energy sources. SMES devices can store electric energy in a superconducting coil without resistive losses, and release their stored energy when required. Compared to other storage technologies, they show a high-density energy, high efficiency and quick power response. Possible applications include load levelling, dynamic stability, transient stability, voltage stability, frequency regulation, transmission capability enhancement, or power quality improvement.

SMES systems store energy as magnetic field created by the flow of direct current in a superconducting coil. Thus, a SMES unit consists of a large superconducting coil at the cryogenic temperature, regulated by a control system. In addition, an AC/DC converter is necessary for connecting with the power system. First generation systems based on LTS have evolved into HTS such as REBCO or YBCO.

\section{HTSG (High Conducting Superconducting Generator)}

Superconductor materials allow high current density, and hence, high magnetic fields, leading to a significant reduction in mass and size for superconducting machine. In addition, the superconducting technology achieves a significant efficiency improvement, especially at partial load. This is particularly relevant to wind power generation since the wind turbines operate mostly at partload conditions. HTSG are also suitable candidates as offshore wind generators, because transport and installation is eased due to a lower volume, in comparison with conventional machines.

\section{SFCLs (Superconducting Fault Current Limiters)}

Fault Current Limiters (FCLs) are used to limit, with high speed, very high currents during faults. Nowadays, with higher penetration rates of renewable energies, shortcircuit current are rising. Thus, FCLs can provide an economical alternative to cope with those currents by installing them at bus-bar coupling or in-line. SFCLs provide one of the most promising solutions for limiting fault currents in power grids by using superconducting materials. Under normal operation, if temperature is maintained within the critical temperature, the device shows no resistance. But for faults exceeding a limit current, the resistance increases and thus, fault current is limited. Hence, compared to other alternatives, they have negligible impedance at normal conditions, fast and effective current limitation within the first current rise and repetitive operation with fast and automatic recovery.

\section{Description of SFCL characteristics}

There have been proposed four main types of SFCLs: resistance type, rectifier-type, saturated-core-type and magnetic-shield-type. Resistive SFCLs add a resistance to the protected circuit during a fault, whereas the rest add an inductance. Moreover, in the rectifier and the saturated core types, the superconductor material does not quench [2].

Nowadays, resistive SFCLs show advantages over the other types for the following reasons:

- They have a simpler structure, smaller size and lower capital cost.

- Resistive SFCLs can not only restrain the fault current, but also consume electrical power of the generators during the fault condition. Thus, transient stability is enhanced.

- They are applicable for both AC and DC currents.

However, resistive SFCLs show slow recovery times and restricted fault limitation.

Those disadvantages are being covered by hybrid-type SFCLs, which consist of a superconducting wire, a fastspeed switch and a current limiting part. They are based on modules which can limit the peak current during the half-cycle period. Thus, the coordination with conventional relays is possible [3]. However, hybrid SFCLs are still being evaluated for commercial use.

Therefore, the present paper focuses on the resistive-type SCFLs, and their operation is analysed in the following lines.

A resistive SFCL is composed of a superconducting cable and a shunt resistance, also called stabiliser resistance, as indicated in Fig. 1. Within critical conditions, resistive 
SFCLs show no resistance for current. During this period, they are operating under a superconducting state. Under faults, current increases highly and so does the resistance of superconductors due to the rise of the superconductor temperature. This stage is commonly called the flux-flow state. Once the superconducting material is fully quenched, the shunt resistance is inserted in the circuit in order to reduce over-voltages. Then, the SFCL operates in normal conducting state. Once the fault is cleared, resistive SFCLs may need a recovery time, during which the element is cooled until it returns to its superconducting state. However, SFCLs can be designed so as to operate immediately after the normal conducting state. Two approaches have been proposed [4]: the constant temperature design and the operational recovery approach.

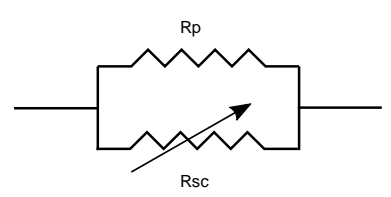

Fig. 1. A resistive SFCL.

The sudden change in the superconductor resistance Rsc is mainly due to the electrical properties of the superconductor material which can be macroscopically simplified into $\mathrm{E}(\mathrm{J})$ power laws, where the electric field depends on the current density and both parameters depend on temperature. Most references are based on [4] for the $E(J)$ characteristic of the superconductor, making use of experimental results of the first SFCL ever installed, made up of Bi2212 materials. As shown in Fig. 2, E(J) characteristic can be divided in three regions limited by curves E1, E2 and E3.

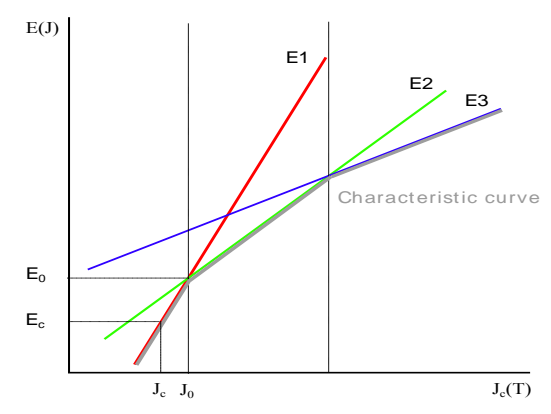

Fig. 2. Characteristic $\mathrm{E}(\mathrm{J})$ curve.

Curve E1 in red represents the superconducting region curve, E2 in green the flux-flow region curve, and E3 in blue the normal conducting region curve. The intersection of the three curves makes up the actual characteristic curve, depicted in gray depending on the current density of each instant.

Hence, as current increases during a fault, the current limiter will go through those three states. Each state is characterised by a power law (Equations (1) to (3)).

$$
E_{1}=E_{c} \cdot\left(\frac{J}{J_{c}(T)}\right)^{\alpha(T)}
$$

$$
\begin{aligned}
& E_{2}=E_{0} \cdot\left(\frac{E_{c}}{E_{0}}\right)^{\beta / \alpha(77 K)} \cdot\left(\frac{J_{c}(77 K)}{J_{c}(T)}\right) \cdot\left(\frac{J}{J_{c}(77 K)}\right)^{\beta} \\
& E_{3}=\rho\left(T_{c}\right) \cdot \frac{T}{T_{c}} \cdot J
\end{aligned}
$$

where $J_{c}$ is the critical current which must be fitted to experimental data; $\alpha$ and $\beta$ depend on material processing conditions, $\rho$ normal resistivity and $T_{c}$ the critical temperature. $\operatorname{Jc}(\mathrm{T})$ and $\rho(\mathrm{T})$ can be approximated as linear functions of temperature by equations (4) and (5).

$$
\begin{aligned}
& J_{c}(T) \approx J_{c}(77 K) \cdot\left(\frac{T_{c}-T}{T_{c}-77 K}\right) \\
& \rho(T) \approx \rho_{c}\left(T_{c}\right) \cdot\left(\frac{T}{T_{c}}\right)
\end{aligned}
$$

In order to include the effect of the magnetic field and based on experimental data, $\alpha=10$ and $\beta=3$ are considered in [4]. Other values can be found in literature based on several experimental results.

During the flux-flow and normal state, power is dissipated and temperature rise versus time can be calculated by (6) [5], where $c$ is the heat capacity per volume. Adiabatic and isotherm conditions are considered. A more complex calculation is given in (7) [6], where $T_{a}$ is the ambient temperature, $Q_{s c}$ the heat dissipated in the superconductor and $\mathrm{Q}_{\text {removed }}$ the heat removed by the coolant.

$$
\begin{aligned}
& \frac{d T}{d t}=E \cdot \frac{J}{c} \\
& T(t)=T_{a}+\int_{0}^{t}\left(Q_{s c}(t)-Q_{\text {removed }}(t)\right) \cdot d t
\end{aligned}
$$

\section{Modelling of resistive SFCLs}

When modelling resistive SFCLs, several perspectives have been proposed in the literature (Table I).

As indicated in Fig. 1, a resistive SFCL is composed of a superconducting cable and a shunt resistance, which reduces over-voltages during quenching. It can also be required to divert part of the fault current after the quench in order to prevent overheating [6]. The shunt resistance can be bonded to the superconductor and/or external. As proposed by Blair et al. in [7], it is often neglected in order to simplify the analysis. Most detailed approaches include a series inductance [8]. In Sung et al. [9], a unit consists of the stabiliser resistance Rns, the superconductor resistance Rnc, and the coil inductance Ln. However, its value in normal condition is very small, and as a consequence, it is often neglected in modeling. Often, a SFCL is composed of several series units (n) in order to reach the specified rating. 
Table I. - Operating principle of resistive SFCLs.

\begin{tabular}{|l|l|}
\hline Sudel & Reference \\
\hline Suir et al. [7]
\end{tabular}

The superconducting materials respond to changes in critical current, critical temperature, and critical magnetic field. Hence, the resistance of the superconducting tapes varies in function of current, temperature and magnetic field.

This section reviews different types of resistive SFCL models described in the literature. On the one hand, simplified and magneto-thermal models are based on one dimension models, and have been developed by simulation packages such as ATP/EMTP, PSCAD/EMTDC and MATLAB/Simulink. On the other hand, for dealing with two and three dimension symmetries, the numerical modelling of SFCL can be based on Finite Element Methods (FEM). Finally, hardware-in-the-loop models are also mentioned in the literature.

\section{A. Simplified models}

Simplified models consider a $\mathrm{R}(\mathrm{t})$ perspective of the superconducting material, i.e. the influence of current, temperature or magnetic field is disregarded for simplicity. Two models have been proposed in the literature: binary models or non-linear loop-up table of resistance values.

Binary models show a negligible resistance in steady-state and a high resistance immediately after a fault occurs. Hence, only superconducting and normal state are considered. However, superconductor materials need a finite time to heat up and develop resistance.

The non-linear look-up table method indicates the SFCL resistance over time based on generic quenching equations. A linear model is presented in [10], but the exponential model in (7), as introduced in [11], is most widely accepted. An alternative to the exponential model in (7) is also presented in [12], all belonging to $\mathrm{S} / \mathrm{N}$ (superconducting-to-normal) transition-type SFCLs.

$R(t)=R_{\max } \cdot\left(1-e^{-t / \tau}\right)$ where $\mathrm{R}_{\max }$ represents the maximum resistance value at the normal state, which is attained $\tau$ seconds after the initiation of a fault.

The quenching process can be triggered at a specific time in simulation or when the instantaneous phase current exceeds a critical current Ic. Thus, the resistance of the SFCL is zero if the phase current is smaller than Ic, while it corresponds to (7) for higher fault currents.

In addition, once the fault has been cleared, the transition from the normal state to the superconducting state of the SFCL is not instantaneous. There is a recovery process, as modelled in [9]. Those models are called N/S (normalto-superconducting) models.

$$
R(t)=\left\{\begin{array}{ll}
0 & \text { if } t<t_{0} \\
R_{\max } \cdot \sqrt{1-e^{-\left(t-t_{0}\right) / \tau}} & \text { if } t_{0} \leq t<t_{1} \\
a_{1} \cdot\left(t-t_{1}\right)+b_{1} & \text { if } t_{1} \leq t<t_{2} \\
a_{2} \cdot\left(t-t_{2}\right)+b_{1} & \text { if } t \geq t_{2}
\end{array}\right\}
$$

$t_{0}, t_{1}$, and $t_{2}$ refer respectively to quenching time, first recovery starting time and second recovery starting time. Response time $t_{1}$ is often considered $2 \mathrm{msec}$ and recovery time $t_{2}$ can be adjusted under the reclosing time in the power system.

Fig. 3 compares the simplified models as mentioned in the present subsection, for a single fault cycle. According to the binary model, the superconducting material in the SFCL has a negligible resistance in steady-state and under faults, resistance increases immediately so as to limit the fault current. Regarding the $\mathrm{S} / \mathrm{N}$ model, an exponential model for the flux-flow state is presented in Fig. 3. On the contrary, once the fault is cleared, the superconductor is supposed to recover its superconducting state without delay. Finally, the N/S model includes an exponential flux-flow state and a linear recovery.

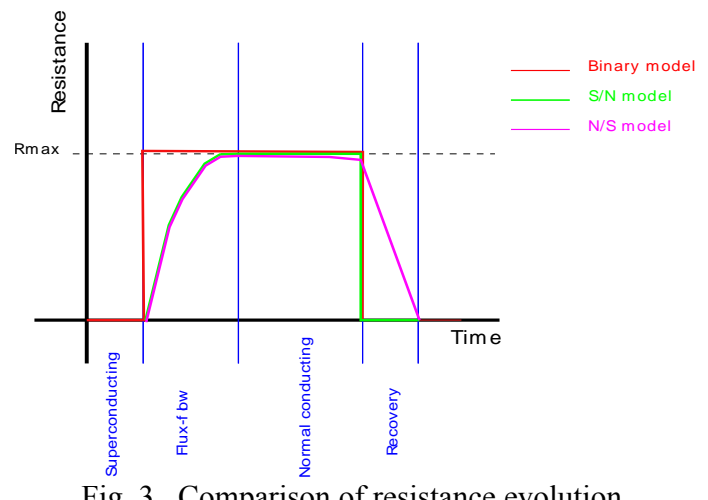

Fig. 3. Comparison of resistance evolution.

\section{B. Magneto-Thermal models}

The evolution of the superconductor resistance is based on E-J power laws. Hence, a $\rho(J, T)$ perspective is considered in thermal models, by using equations (10)(12). 
$\rho=\left\{\begin{array}{ll}0 & \text { if } J<J_{c}, \mathrm{~T}<T_{c} \\ \rho_{c} \cdot \frac{J}{J_{c}} & \text { if } J>J_{c}, \mathrm{~T}<T_{c} \\ \rho_{\text {HTS }} & \text { if } T>\mathrm{T}_{c}\end{array}\right\}$

where:

$\rho_{c}=\frac{E_{c}}{J_{c}}$

$J_{c}=J_{c}(77 K) \cdot \frac{\left(T_{c}-T\right)}{T_{c}-77 K}$

In addition, more detailed models include heat transfer capacity expressions of the superconducting materials. Some references include the shunt resistance. However, the classic magneto-thermal models can result into complicated calculations and long simulation times. Therefore, simplified magneto-thermal models, based on empirical results, have been proposed in [13], [14], or [15].

Based on the aforementioned thermal models, some references propose a $\mathrm{R}(\mathrm{I}, \mathrm{T})$ perspective, considering a V-I characteristic, given that voltage $\mathrm{V}$ is related to electric field $\mathrm{E}$ and current $\mathrm{I}$ to current density J. According to [16], once the critical current is reached, resistance increases non-linearly as indicated in (13).

$R_{1}=\frac{d V}{d I}=\frac{n \cdot V_{c} \cdot I^{n-1}}{I_{c}^{n}}$

where $\mathrm{n}$ is the resistive transition index, $\mathrm{I}_{\mathrm{c}}$ the critical current and $\mathrm{V}_{\mathrm{c}}$ the critical current.

Then, after reaching the maximum value of resistance, there is a linearly exponential decay in (14) corresponding to the normal conducting state.

$R_{2}=R_{\max } \cdot e^{-t_{2} / \tau}$

where $R_{\max }$ is the maximum value of resistance, $t_{2}$ is the start of the decay interval and $\tau$ the time constant.

Finally, the superconducting material returns to the superconducting state with some slope, as indicated by (15).

$R_{3}=R_{c}-\frac{R_{c}}{m} \cdot\left(t_{2}-t_{3}\right)$

where $R_{c}$ is the critical value of resistance at which the full power cooling is applied, $\mathrm{m}$ the negative slope of the recovery, and $t_{3}$ is the start of the recovery interval.

\section{Finite element analysis models}

Several models have been developed by detailed finite element analysis, mainly for inductive-type SFCLs [17], [18], [19], but also for resistive-type [20], [21], [22]. However, FEA models require specialised software, such as Comsol, Magnet or Flux2D. Model implementation can result into a complicated process and simulation times are often long. But simulation results from FEA models can be used to validate other models with high accuracy [23].

\section{Hardware-in-the-loop models}

Several references use Real-Time Simulation (RTS) methodology with Power-Hardware-In-the-Loop (PHIL) capabilities [24], [25], [6]. Thus, power amplifiers pass the output current from a circuit simulator through the superconductor wire under test. The RTS-PHIL enables to include the actual transient reaction of the hardware under study without any complicated numerical model, while the power system circuit can be simulated.

\section{Conclusions}

The present paper has reviewed the main applications of superconducting materials in the power system, mainly focusing on SFCLs. Then, several modelling approaches of SFCLs have been presented, focusing on resistive-type limiters because of size, cost and product maturity.

Superconducting materials go through transient states when submitted to fault currents. Therefore, modelling is complex. As a consequence, some theoretical assumptions have to be considered in order to analyse the operation of a resistive SFCL. One dimension models are commonly adopted for studying superconductivity and include simplified models as well as magneto-thermal models. Two and three dimension models, which are based on FEM, show high accuracy results although the required simulation times are long. Hardware-in-the-loop models exhibit the real transient response of the superconductor devices with no numerical model.

\section{Acknoledgements}

The authors thank the support from the Basque Government (projects ETORTEK IE14-389 and ELKARTEK HVDCLINK) and the University of the Basque Country UPV/EHU (project EHUA15/25).

\section{References}

[1] A. Morandi, S. Brisigotti, G. Grasso, and R. Marabotto, 'Conduction Cooling and Fast Recovery in -Based DC Resistive SFCL', IEEE Trans. Appl. Supercond., vol. 23, no. 5, pp. 5604409-5604409, Oct. 2013.

[2] S. Eckroad, 'Superconducting Fault Current Limiters', Electric Power Research Institute EPRI, Palo Alto, California, 1017793, 2009.

[3] G.-H. Lee, K.-B. Park, J. Sim, Y.-G. Kim, I.-S. Oh, O.-B. Hyun, and B.-W. Lee, 'Hybrid Superconducting Fault Current Limiter of the First Half Cycle Non-Limiting Type', IEEE Trans. Appl. Supercond., vol. 19, no. 3, pp. 1888-1891, Jun. 2009.

[4] W. Paul, M. Chen, M. Lakner, J. Rhyner, D. Braun, W. Lanz, and M. Kleimaier, 'Superconducting Fault Current Limiter Applications, technical and economical benefits, simulations and test results', presented at the Session CIGRE 2010, Paris, France.

[5] L. Ye and K.-P. Juengst, 'Modeling and simulation of high temperature resistive superconducting fault current limiters', IEEE Trans. Appl. Supercond., vol. 14, no. 2, pp. 839-842, Jun. 2004.

[6] J. Langston, M. Steurer, S. Woodruff, T. Baldwin, and J. Tang, 'A generic real-time computer Simulation model for 
Superconducting fault current limiters and its application in system protection studies', IEEE Trans. Appl. Supercond., vol. 15, no. 2, pp. 2090-2093, Jun. 2005.

[7] S. M. Blair, C. D. Booth, and G. M. Burt, 'Current -Time Characteristics of Resistive Superconducting Fault Current Limiters', IEEE Trans. Appl. Supercond., vol. 22, no. 2, pp. 5600205-5600205, Apr. 2012.

[8] S. Nemdili and S. Belkhiat, 'Modeling and Simulation of Resistive Superconducting Fault-Current Limiters', J. Supercond. Nov. Magn., vol. 25, no. 7, pp. 2351-2356, Jun. 2012.

[9] B. C. Sung, D. K. Park, J.-W. Park, and T. K. Ko, 'Study on a Series Resistive SFCL to Improve Power System Transient Stability: Modeling, Simulation, and Experimental Verification', IEEE Trans. Ind. Electron., vol. 56, no. 7, pp. 2412-2419, Jul. 2009.

[10] H. K. (Criepi) and S. Torii(Criepi), 'Setting method of parameters for $\mathrm{SN}$ transition fault current limiter into $6.6 \mathrm{kV}$ distribution system', J. Phys. Conf. Ser., vol. 97, no. 1, p. 012322, 2008.

[11] I. Muta, T. Doshita, T. Nakamura, T. Egi, and T. Hoshino, 'Influences of superconducting fault current limiter (SFCL) on superconducting generator in one-machine double-line system', IEEE Trans. Appl. Supercond., vol. 13, no. 2, pp. 2206-2209, Jun. 2003.

[12] Y. Ye, L. Xiao, H. Wang, and Z. Zhang, 'Research on Resistor Type Superconducting Fault Current Limiter in Power System', in Transmission and Distribution Conference and Exhibition: Asia and Pacific, 2005 IEEE/PES, 2005, pp. 1-6.

[13] P. E. Sutherland, 'Analytical model of superconducting to normal transition of bulk high Tc superconductor BSCCO-2212', IEEE Trans. Appl. Supercond., vol. 16, no. 1, pp. 43-48, Mar. 2006.

[14] F. Roy, B. Dutoit, F. Grilli, and F. Sirois, 'Magneto-Thermal Modeling of Second-Generation HTS for Resistive Fault Current Limiter Design Purposes', IEEE Trans. Appl. Supercond., vol. 18, no. 1, pp. 29-35, Mar. 2008.

[15] P. J. C. Branco, M. E. Almeida, and J. A. Dente, 'Proposal for an RMS thermoelectric model for a resistive-type superconducting fault current limiter (SFCL)', Electr. Power Syst. Res., vol. 80, no. 10, pp. 1229-1239, Oct. 2010.

[16] Elizaveta Egorova, Himanshu Bahirat, Bruce A. Mork, Warren F. Perger, and Matthew Holcomb, 'EMTP-ATP Modeling of a Resistive Superconducting Fault Current Limiter', presented at the International Conference on Power Systems Transients (IPST2013, Vancouver, Canada, 2013.

[17] J. Y. Zhang, Z. Q. Wei, H. Hong, W. Z. Gong, and Y. Xin, 'Electromagnetic design of saturated iron core SFCL', in 2011 International Conference on Applied Superconductivity and Electromagnetic Devices (ASEMD), 2011, pp. 305-308.

[18] H. Heydari, A. A. Abrishami, and M. M. Bidgoli, 'Comprehensive Analysis for Magnetic Shield Superconducting Fault Current Limiters', IEEE Trans. Appl. Supercond., vol. 23, no. 5, pp. 5604610-5604610, Oct. 2013.

[19] M. Majka, J. Kozak, S. Kozak, G. Wojtasiewicz, and T. Janowski, 'Design and Numerical Analysis of the $15 \mathrm{kV}$ Class Coreless Inductive Type SFCL', IEEE Trans. Appl. Supercond., vol. 25, no. 3, pp. 1-5, Jun. 2015.

[20] S. Kozak, T. Janowski, B. Kondratowicz-Kucewicz, J. Kozak, and G. Wojtasiewicz, 'Experimental and numerical analysis of energy losses in resistive SFCL', IEEE Trans. Appl. Supercond., vol. 15, no. 2, pp. 2098-2101, Jun. 2005.

[21] J. B. Na, Y. J. Kim, J. Y. Jang, K. S. Ryu, Y. J. Hwang, S. Choi, and T. K. Ko, 'Design and Tests of Prototype Hybrid Superconducting Fault Current Limiter With Fast Switch', IEEE Trans. Appl. Supercond., vol. 22, no. 3, pp. 5602604-5602604, Jun. 2012.

[22] Y. J. Kim, D. K. Park, S. E. Yang, W. C. Kim, M. C. Ahn, Y. S. Yoon, N. Y. Kwon, H. Lee, and T. K. Ko, 'Analytical Design Method of High-Tc Coated Conductor for a Resistive Superconducting Fault Current Limiter Using Finite Element Method', IEEE Trans. Appl. Supercond., vol. 20, no. 3, pp. 11721176, Jun. 2010

[23] Y. Chen, S. Li, J. Sheng, Z. Jin, Z. Hong, and J. Gu, 'Experimental and Numerical Study of Co-ordination of Resistive-Type Superconductor Fault Current Limiter and Relay Protection', J. Supercond. Nov. Magn., vol. 26, no. 11, pp. 32253230, Apr. 2013.

[24] O. Naeckel, J. Langston, M. Steurer, F. Fleming, S. Paran, C. Edrington, and M. Noe, 'Power Hardware-in-the-Loop Testing of an Air Coil Superconducting Fault Current Limiter
Demonstrator', IEEE Trans. Appl. Supercond., vol. 25, no. 3, pp. 1-7, Jun. 2015.

[25] L. Graber, T. Chiocchio, J. Kvitkovic, S. Pamidi, M. Steurer, and A. Usoskin, 'Finite Element Model of a Superconducting Fault Current Limiter Calibrated by Hardware-in-the-Loop Measurements', IEEE Trans. Appl. Supercond., vol. 24, no. 3 , pp. 1-5, Jun. 2014. 